\title{
POLYCHLORINATED BIPHENYLS AFFECT APOPTOSIS OF PITUITARY CELLS THROUGH EXTRINSIC AND INTRINSIC PATHWAYS
}

Francesco Raggi ${ }^{1}$, Dania Russo ${ }^{1}$, Claudio Urbani ${ }^{1,2}$, Daniele Cappellani ${ }^{1}$, Luca Manetti ${ }^{1}$, Chiara Sardella ${ }^{1}$, Isabella Lupi ${ }^{1}$, Ilaria Scattina ${ }^{1}$, Luca Tomisti ${ }^{1}$, Claudio Marcocci ${ }^{1}$, Enio Martino ${ }^{1}$, Fausto Bogazzi $^{1}$

${ }^{1}$ Department of Clinical and Experimental Medicine, Section of Endocrinology, University of Pisa, Pisa, Italy; ${ }^{2}$ Sant'Anna School of Advanced Study, Pisa, Italy

\section{Introduction and objectives}

Polychlorinated biphenyls (PCBs) are environmental pollutants that modulate endocrine functions, induce tumorigenesis, and regulate apoptosis in several tissues; however, their effects on the apoptosis of pituitary cells is unknown. The aim of this study was to evaluate the PCBs influence on the apoptosis of normal pituitary cells and elucidate the molecular mechanisms involved.

\section{Methods}

Cell cultures and chemicals treatment Primary cell cultures from C57B1/6J mouse pituitary glands were exposed to a PCBs mixture (Aroclor 1254) or dioxin-like (PCB 77, PCB 126) or non-dioxin-like (PCB 153, PCB 180) congeners at $10 \mu \mathrm{M}$ for $24 \mathrm{~h}$. Cisplatin (32 $\mu \mathrm{M})$ was used as a positive control.

Apoptosis analysis Apoptosis was assessed by Annexin V staining, DNA fragmentation and TUNEL assay. The expression and activity of intrinsic and extrinsic pathways were evaluated by Western blot and ELISA assay, respectively. Thyroid hormone receptor (TR), aryl-hydrocarbon receptor (AhR), and CYP1A1 antagonists were used to examine the mechanisms of PCBs action.

\section{Results}

The effect of PCBs on the apoptosis of pituitary cells. The early and late phase of pituitary cells apoptosis was raised by Aroclor 1254 or PCB 180, was almost suppressed by PCB 153 or left unaltered by the dioxin-like congeners PCB 126 and PCB 77.

(Fig.1A) Representative flow cytometric scatter plots of Annexin V staining assay: the right bottom quadrant of each plot contains the early apoptotic cells (Annexin V-FITC positive/PI negative). (Fig.1B) Percentages of annexin V-FITC positive cells in the different treatments. (Fig.1 C and D) DNA fragmentation and TUNEL assay in the late

phase of cells apoptosis. (A.U., arbitrary units)

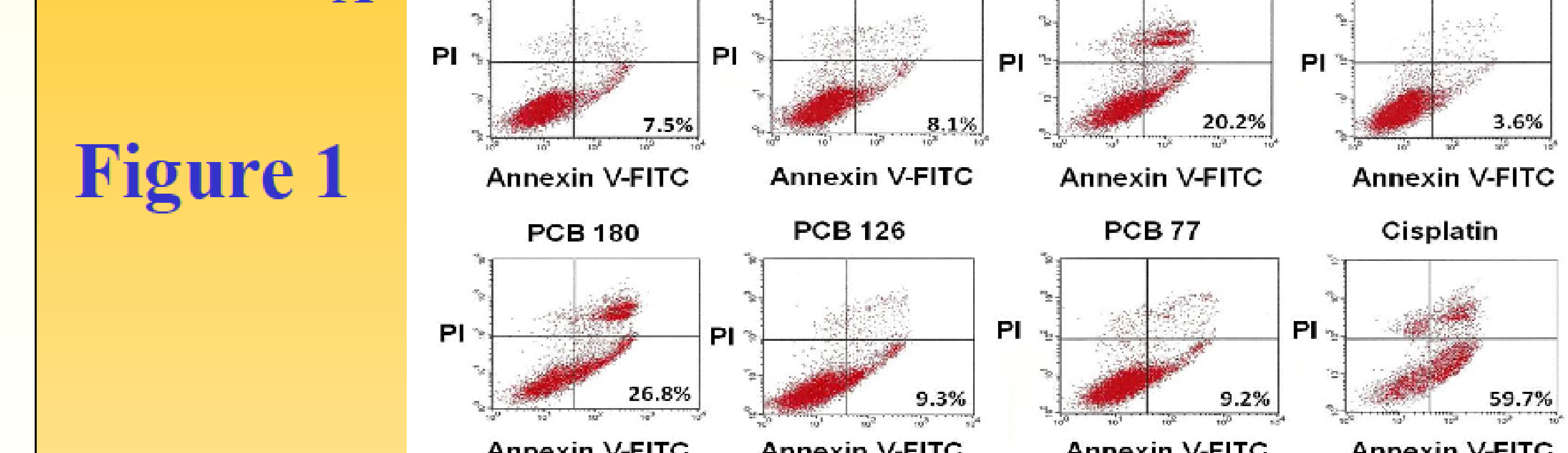

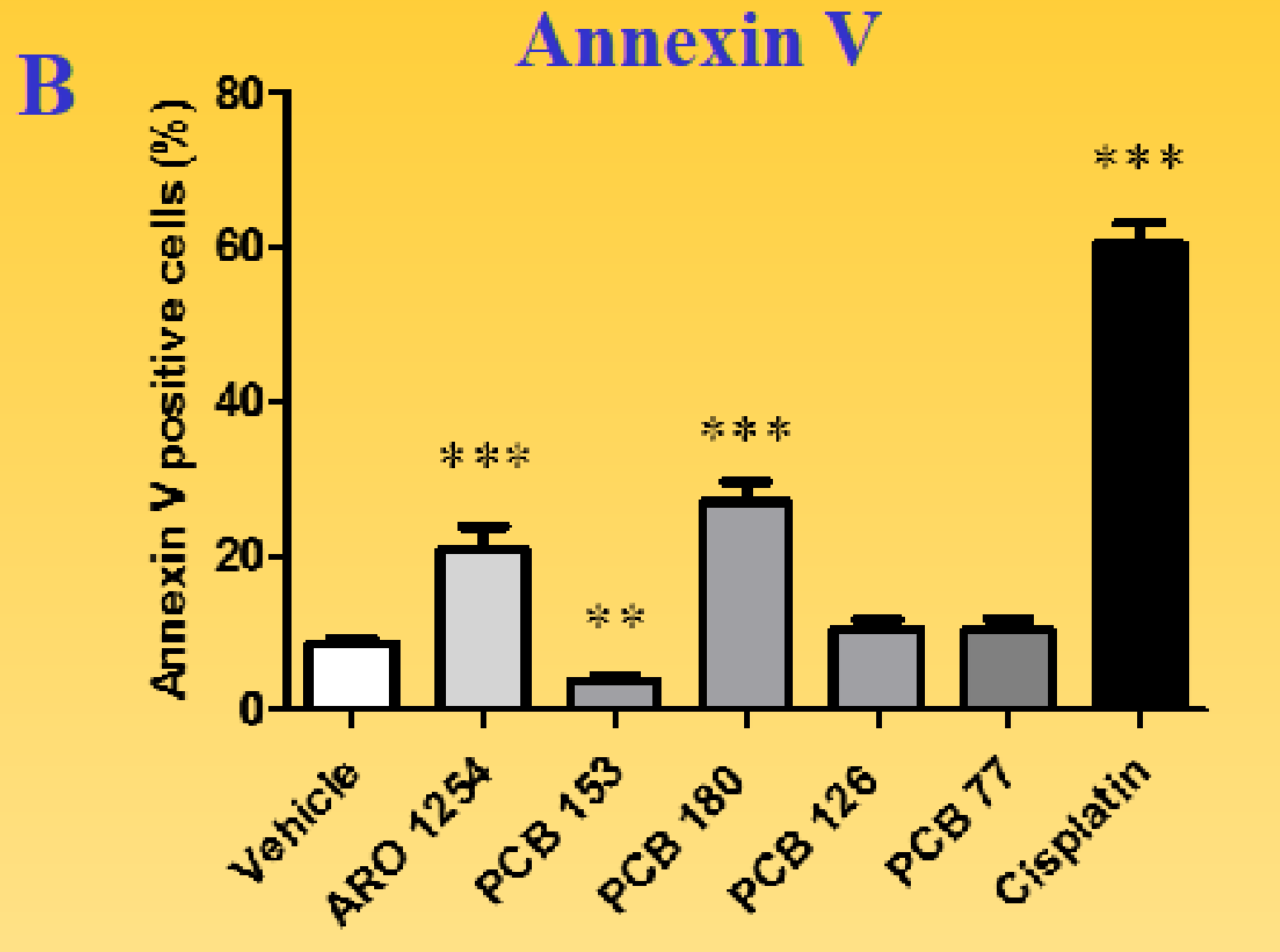

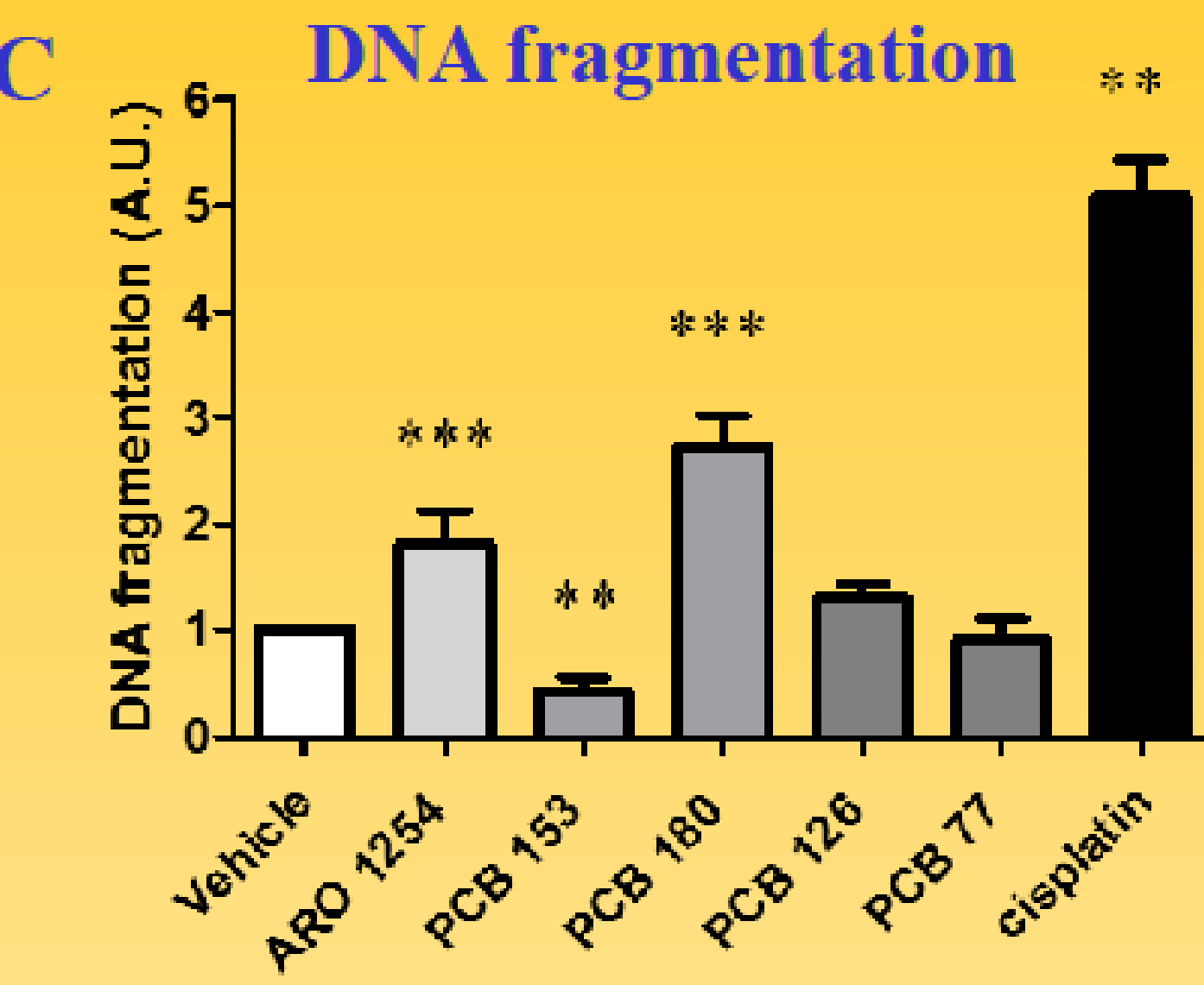

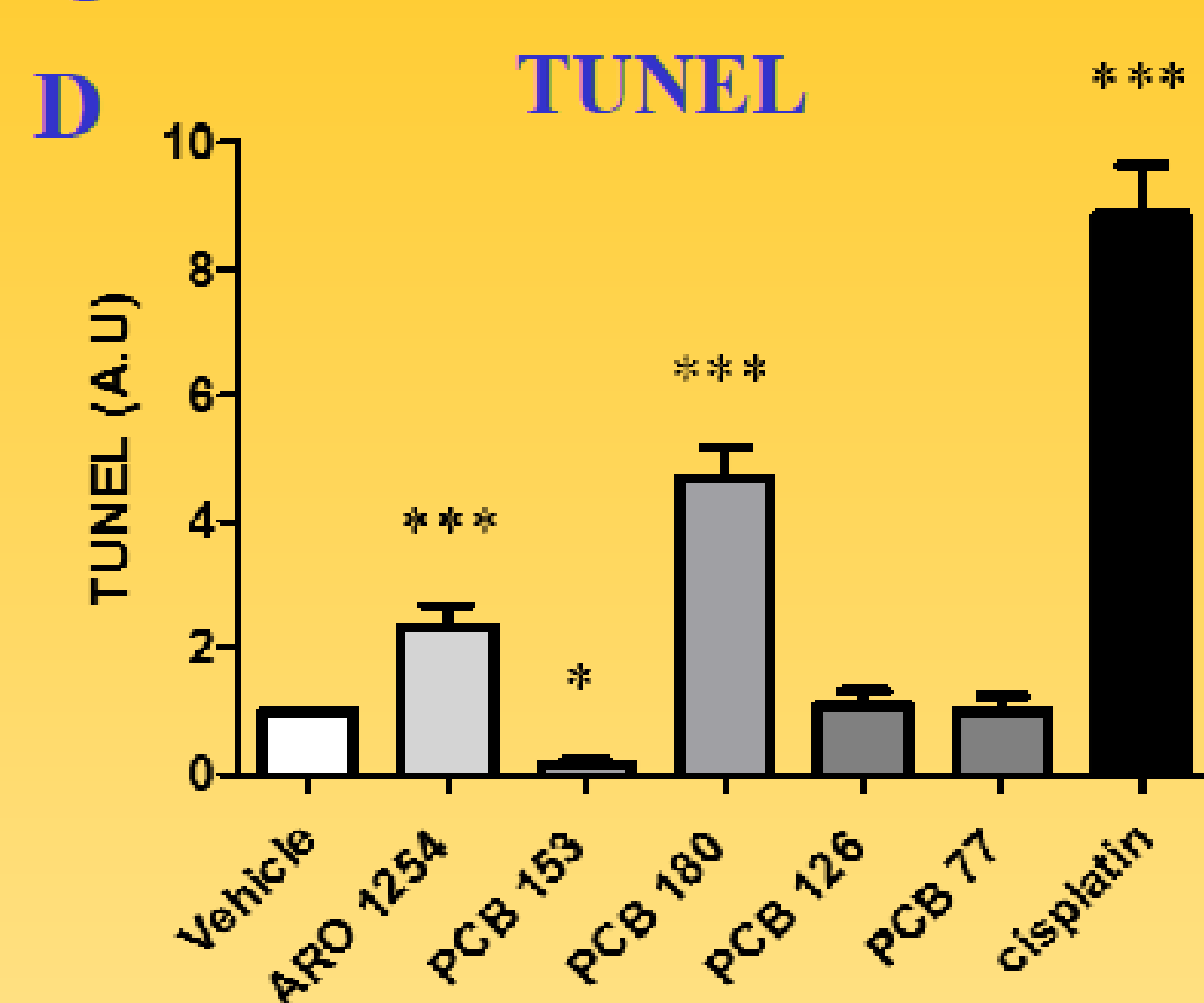

The effect of PCBs on the extrinsic and intrinsic apoptosis pathway: The expression or activity of the final effector of apoptosis, i.e. caspase-3, changed in relation to the change in apoptosis during the exposure to a mixture of PCBs or individual congeners (Fig.2A). PCB 180 influenced the extrinsic pathway by an increase in the expression or activity of caspase-8 (Fig.2B),TNF- $\alpha$ (Fig.2D), TRAIL(Fig.2E) and TRADD (Fig.2G). In contrast, PCB 153 reduced the expression or activity of caspase-8 (Fig.2B), TNF- $\alpha$ (Fig.2D), FAS-L (Fig.2F), TRADD (Fig.2G), and FADD (Fig.2H). In addition, PCB 153 affected the intrinsic apoptosis through a decrease in the expression or activity of caspase-9 (Fig.2C), by an increase of PI3K-AKT (Fig.2 I and L), and a reduction of the p38-p53 (Fig.2 M and N) pathways.

Figure 2

$$
\text { A }
$$

25
20.

年. 1.5.


D
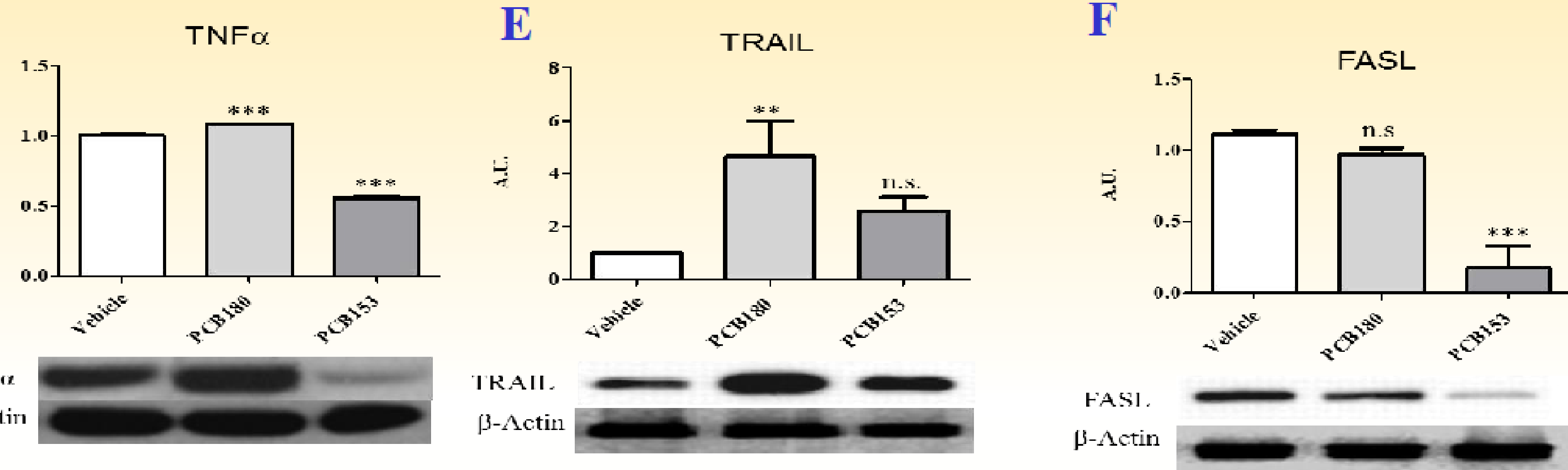

G

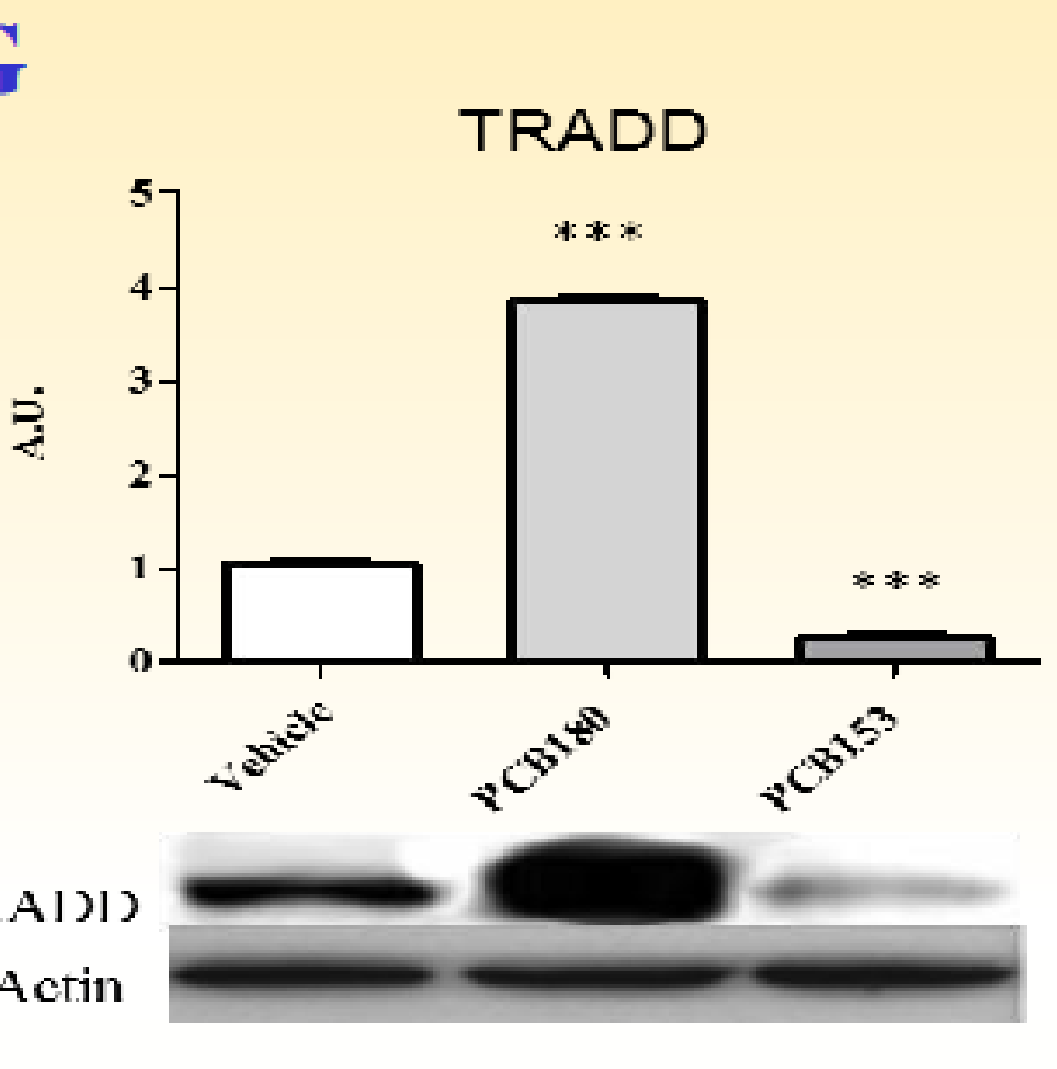

西
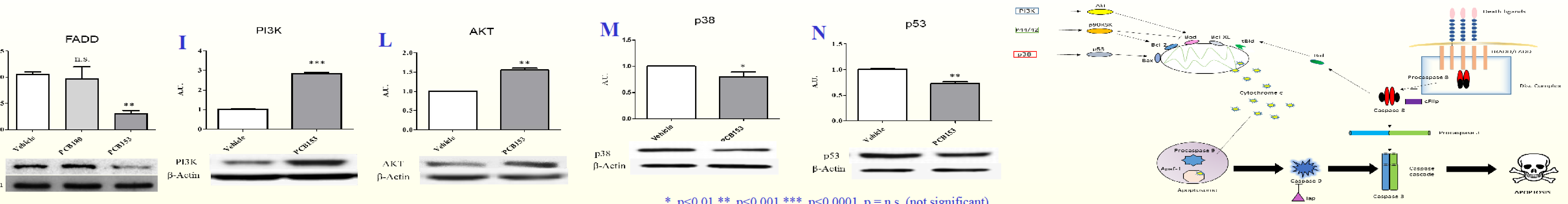

The effect of PCBs on the apoptosis of pituitary cells involved TR, AhR or CYP1A1 receptor and was associated with a reduction in cell proliferation: primary pituitary cells were pretreated with a TR antagonist (1-850), or an AhR inhibitor, $\alpha$-Naphthoflavone ( $\alpha$-NF) or a CYP1A1 inhibitor, 7-Hydroxyflavone (HF). The antiapoptotic phenotype of PCB 153 was counteracted by TR or CYP1A1 antagonists (Fig.3 A and C), whereas the pro-apoptotic effect of PCB 180 was prevented by an AhR antagonist (Fig.3B). As a positive control to the induction of TR-, AhR- or CYP1A1-mediated apoptosis, 3,3',5 triiodothyronine (T3), 2,3,7,8-Tetrachlorodibenzo-p-dioxin (TCDD), and Cycloheximide (CHX) were used. The apoptosis induced by Aroclor 1254 or PCB 180 was associated with a reduction in cell proliferation, whereas the decreased apoptosis due to PCB 153 increased cell proliferation by 30\%. (Fig.3D). Cell proliferation was evaluated by measuring the cellular uptake of 5-bromo-2'-deoxyuridine. The culture medium, supplemented with $10 \%$ fetal bovine serum and Colchicine $(1 \mu \mathrm{M})$ were used as a positive or negative control, respectively.

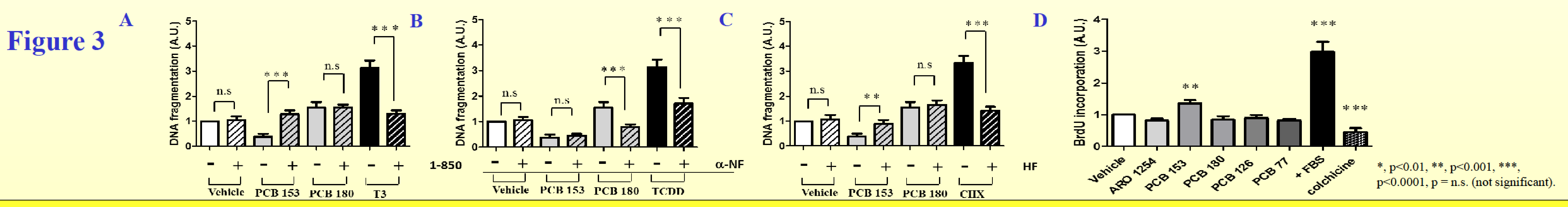

\section{Conclusions}

Non-dioxin-like PCBs may modulate apoptosis and the proliferation rate of pituitary cells having pro- or anti-apoptotic effects depending on specific congeners. 